\title{
Commission 51: Bioastronomy - Search for Extraterrestrial Life
}

\author{
PRESIDENT: Karen Meech \\ VICE-PRESIDENT: Alan Boss \\ ORGANIZING COMMITTEE: Cristiano Cosmovici, Pascale \\ Ehrenfreund, David Latham, David Morrison, and Stephane Udry
}

Keywords. search for extra-terrestrial intelligence, planet formation, extrasolar planets, evolution of advanced life, habitable zones

\section{Bioastronomy Triennial Meetings}

Historically, there have been two main groups dealing with the investigation of extraterrestrial life and habitable worlds. The first is IAU Commission 51, composed of astronomers, physicists and engineers who focus on the search for extrasolar planets, formation and evolution of planetary systems, and the astronomical search for intelligent signals. The second group, the International Society for the Study of the Origin of Life (ISSOL), is composed largely of biologists and chemists focusing research on the biogenesis and evolution of life on Earth and in the solar system. There are now a variety of international organizations dedicated to this field, and this triennium has seen the beginnings of coordination and interaction between the groups through the Federation of Astrobiology Organizations, FAO.

The series of previous Bioastronomy meetings have played an important role in integrating the broader interests and techniques of both astronomy and biology to understand the origin and evolution of living systems in the universe, and to generating a context for exploration in our solar system and in extrasolar planetary systems. These conferences provide an opportunity for astronomers, biologists, geologists, planetary scientists and those from other disciplines to meet and discuss research of mutual interest for addressing the question of the origin and evolution of life on this planet and elsewhere in the universe. The first International Bioastronomy Conferences were visionary in tackling problems that many scientists felt were premature to attack, given our knowledge at the time. However, these conferences are increasingly being held in a world where many others have begun to realize that these seemingly impenetrable questions are becoming amenable to at least provisional answers.

Active involvement by the IAU in these meetings began at the General Assembly in Montreal in 1979, when a Joint Session was held on Strategies for the Search for Life in the Universe. After the establishment of the commission in 1982, the Bioastronomy meetings were held every 3 years beginning in 1984 (1984, IAU Symp. 112, Boston, USA; 1987 IAU Colloq. 99, Balston, Hungary; 1990, Val Cenis, France; 1993, Santa Cruz, USA; 1996 IAU Colloq. 161, Capri, Italy; 1999, Hawaii, USA; 2002 IAU Symp. 213, Hamilton Island, Australia; and 2004, Reykjavik, Iceland).

\subsection{Bioastronomy 2004: Habitable Worlds}

Iceland was selected as the 2004 meeting site (July 12-16, 2004) because of its astrobiologically unique environment. Iceland boasts a unique sub-arctic ecosystem, at the separating boundary of the Eurasian and North American continental plates. Subglacial Icelandic volcanic eruptions and deposits in Antarctica help us to understand the ice/volcano interaction both on earth and other astrobiologically important environments in the solar system. This venue provided a rich and stimulating environment for the meeting topic of "Habitable Worlds" and provided the opportunity to visit scientifically relevant sites: newly formed volcanic islands, sub-arctic 
conditions, thermal springs, and mid-ocean ridges. Bioastronomy 2004 resulted in another major step along our way to answering the fundamental questions of how does life begin and evolve, and does life exist elsewhere in the universe?

Thorsteinn Thorsteinsson, of the National Energy Authority in Iceland, was the Local Organizing Chair for the meeting, and Alan Boss (Carnegie) was the Scientific Organizing chair. The LOC consisted of an interdisciplinary group of 11 scientists, 10 locally from Iceland, and the Commission 51 president. The SOC had 15 international members from diverse backgrounds. The meeting would not have been possible without the generous support from a large number of sponsors, including grants from the National Science Foundation (from the Planetary Astronomy Program in the Astronomy Division and from the Biology Directorate), from the NASA Planetary Astronomy program and from the NASA Astrobiology program. The LOC chair was also successful in soliciting meeting funding from the Icelandic government (from the Prime Minister, the Finance Minister, from the Environmental Ministry and from the Icelandic Coast Guard), as well as in-kind donations from the Orkustofnun, the Icelandic Astronomical Society, Icelandic Centre for Research and the Geoscience Society of Iceland. In addition to using these funds for general meeting support, we were able to provide substantial funding to 24 participants.

A total of 230 abstracts from 212 authors were accepted to the meeting in the following categories: Extrasolar Habitable Worlds; Origin \& Evolution of Terrestrial Life; Life Elsewhere in the Solar System; Life in Extrasolar Planetary Systems; Intelligent Life Beyond the Solar System; and Other. With nearly 100 invited and contributed talks, Bioastronomy 2004 presented a rich smorgasbord of scientific work on the origin, evolution, and prevalence of life in the universe.

The conference opened with an evening program in Reykjavik City Hall on July 11 sponsored by the City of Reykjavik. All of the conference talks were delivered in plenary sessions, with 4 sessions per day led by a 45 minute invited talk, and followed by 15 minute contributed talks. A dedicated poster session with refreshments was held Monday evening, and the posters were available for display throughout the week. A special event was held at the University of Iceland for the student and post doctoral participants in order to foster networking between the younger members of our community. A midweek fieldtrip and 3 post-conference field trips introduced the participants to the rich cultural, geological and astrobiological histories of Iceland. Three public talks were invited from SOC members on the topics of the Origin, history and evolution of water in the universe, Life under ice, and Looking for Earths in nearby solar systems. A midweek teacher workshop was organized for 15 Icelandic science teachers to educate them about astrobiology and to foster possible collaborations in Education and Outreach.

\subsection{Future Bioastronomy Meetings}

Held in years without an IAU General Assembly, originally the Bioastronomy series of meetings was out of phase with meetings hosted by ISSOL. However, in 1992, ISSOL changed the dates of their triennial meetings to avoid the Olympics and they began to hold their meetings in the same year as the Bioastronomy meetings, usually close in time to the Bioastronomy meetings, but not typically close geographically. Beginning in 2001, NASA Ames has hosted a large interdisciplinary meeting every 2 years called AbSciCon. In an attempt to consolidate meetings, and establish a richer interchange of ideas, a small group of people representing various entities within the FAO met to develop a plan for future meetings. This meeting was held at UCLA on September 2, 2004, hosted by ISSOL Board member William Schopf, and attended by representatives from ISSOL (Antonio Lazcano), IAU C51 (Karen Meech), AbSciCon (Lynn Rothschild), NAI (Rose Grymes, also representing the FAO, and the European Eso/Astrobiology Network Association (EANA)). At this meeting a plan for coordinating the Bioastronomy, ISSOL and AbSciCon meetings leading up to a World Astrobiology Congress in 2010 was laid out, and as a result Commission 51 is working with ISSOL in an attempt to hold concurrent meetings in 2008 in Florence, Italy, with a couple of days of joint sessions. 


\section{Science Highlights}

This reporting period, 2002-2005, has seen some exciting developments in the area of Bioastronomy. NASA has had a successful encounter at Titan and NASA and ESA have spacecraft in orbit around Mars, searching the entire surface for hints of water, and NASA has returned to the surface of Mars with rovers that have yielded persuasive evidence for the presence of water on Mars. NASA and ESA have spacecraft that visited or were launched on their way to several comets, frozen repositories of the most primitive molecules that formed the planets of our Solar System and may have carried prebiotic chemicals to the terrestrial planets. NASA and ESA continue to plan for space telescopes capable of detecting Earth-like planets around the closest stars. Here on Earth, research in laboratories and in the field has continued to explore the possibilities for the origin, evolution, and persistence of life in seemingly hostile environments.

\subsection{SETI}

There are currently 5 ongoing radio SETI surveys, including META II (1990-), Project Argus (1995-), SERENDIP IV (1996-), So. SERENDIP (1998-), and SETItalia (2000-). In addition, there are 7 ongoing targeted surveys, including Project Phoenix (radio; 1998-) and 5 optical SETI programs: Harvard OSETI (1998-), Princeton (2001-), Berkeley (1998-), Lick (2001-), and OZ Seti (2000-) at the University of Western Sydney and 2 large amateur SETI organizations (the SETI League and project BAMBI). Finally, there is also the unique distributed SETI search of SETI@home, designed as a screen saver which takes advantage of unused personal computer cpu cycles to analyze data from the SERENDIP IV project. In addition an exciting new instrument is currently under development, called the Allen Telescope Array (ATA, formerly the 1hT array), a joint effort by the SETI Institute and the University of California, Berkeley. This facility will be simultaneously used for SETI and radio astronomy research. The facility will involve 350 twenty-foot diameter dishs located 250 miles northeast of San Francisco. Currently three dozen dishes are installed and by spring 200642 antennas will be working and it will embark upon an Inner Galactic plane survey. With our present radio extraterrestrial intelligence, and noted that with our present radio communications technology (i.e., the Arecibo radar transmitter and radio telescope) we are able to communicate with another civilization with the same degree of technological development living around stars only as far away as 100 light years, a very small region of our galaxy. However, with the advent of the ATA and the proposed Square Kilometer Array (SKA), it is projected that it is likely that within our lifetimes, we will know the answer to the question of whether intelligent life is abundant in the universe or not.

\subsection{Extrasolar Planet Discoveries}

The past three years have been an exceptionally productive period for the discovery and characterization of extrasolar planetary systems. Perhaps $25 \%$ of nearby stars seem to be good analogues for our planetary system in that they appear to have a long-period Jupiter-like planet, but no evidence for short-period Jupiter-like planets that would interfere with the existence of a habitable Earth-like planet on a short-period (1 year) orbit. M dwarf stars, the most plentiful in the galaxy, are also now known to contain planetary systems, increasing the number of possible abodes for habitable planets. The discovery of the fourth pulsar planet, orbiting a binary system in the M4 globular cluster, showed that Jupiter-mass planets can form even in regions of extremely low metallicity compared to the solar neighborhood. Further discoveries may be found in the report from the IAU Working Group on Extrasolar Planets.

\subsection{Habitable Worlds}

Star formation takes place within molecular clouds, and understanding their formation, and evolution during star formation is critical toward an understanding of the formation of habitable worlds. There have been advances in both the theory of cloud origins, advances in dynamics and in the observations of disks. Considerable progress is also being made in understanding the accretion disks around sun-like stars in the context of low long the gas-rich disks survive, what their transitions from the gas-rich state, to the post accretionary debris disk state are, and what the range in properties of the disks are. In particular, there has been tremendous advance 
in our ability to resolve disks observationally in the near and mid-infrared as well as in the sub-millimeter. While a consensus is emerging concerning the early evolution of the disks and the characterization of the older debris disks, research is now focusing on the transition between these extremes which is believed to occur during the epoch of planet formation.

\subsection{Evolution in the Solar System - Habitable Environments and Pre-biotic Chemistry}

Cassini Mission to Saturn and Titan - The Huygens probe underwent a soft landing on the surface of Titan on 14 January 2005, encountering a surface at 94K with liquid methane and rocks composed of dirty ice. Surface features indicative of Earth-like processes such as precipitation and fluid flow were evident. Nitrogen was found to be the dominant gas in the upper atmosphere, and methane increased in abundance in the lower atmosphere, with the levels changing over time, implying a release of methane from the surface or subsurface. Work is actively continuing to understand the internal structure and composition of Titan and how it relates to the primordial atmosphere of Earth.

Mars Missions - There are currently 5 active missions at Mars: Mars Global Surveyor (MGS), Mars Odyssey, Mars Exploration Rovers (MER), Mars Reconnaissance Orbiter (MRO; which will reach Mars in Mar. 2006 and begin its primary mission in Nov. 2006), and Mars Express. Since landing in early 1994 the MER mission has provided strong evidence for a watery past on Mars. However, because of the widespread presence of olivine, which weathers quickly in the presence of water, the Mars environment must be quite dry overall. The amount of frozen water found by $\mathrm{MRO}$ in some regions is too large to be in equilibrium with the atmosphere, suggesting that the planet may be going through a period of climate change.

The Deep Impact Mission - NASA's eighth Discovery mission, Deep Impact was launched on January 12, 2005, and successfully impacted comet 9P/Tempel 1 on July 4, 2005. This mission was designed to take a look at primordial solar system materials, by digging underneath the evolved surface of a comet nucleus. Constrained by limitations on budget for this class of mission, Deep Impact was a fast flyby which had imaging and spectroscopic capability. To complement the spacecraft, an unprecedented world-wide coordinated observing effort involving over 50 observatories and nearly 800 nights of telescope time since 1997 contributed a major component to this mission. Spectacular images from the encounter showed a different appearance from that of the other comets visited by spacecraft, indicating that at least superficially, comet nuclei are very diverse. Tempel 1 clearly showed features which are consistent with impact craters that may be associated with older regions. Both the ground and spacecraft data showed that while the impact induced some activity, it was short-lived, and behaved like a natural comet outburst. However there were changes in chemistry (with an increase in organic material seen) and dust grain size and composition as a result of the impact, and once fully analyzed, this should give us insight into the type of material delivered to the pre-biotic Earth. This information is also invaluable for the ESA Rosetta mission, enroute to comet Churyumov Gerasimenko for arrival in summer 2014.

Karen J. Meech, and Alan Boss

President/Vice President of the Commission 